\title{
Improved MLT and Its Applications to Solar Models
}

\author{
Y. Li and J. Y. Yang \\ Yunnan Observatory, NAOCAS, P.O. Box 110, Kunming, 650011 China
}

\begin{abstract}
Based on an improved MLT that takes the anisotropy and dissipation of the turbulence into account, we found that the dissipation of the turbulence in the solar convection zone should be fairly strong, while the turbulence anisotropy plays a less significant role.
\end{abstract}

\section{Improved MLT Based on Turbulent Convection}

The most uncertainty in present solar models lies in the treatment of turbulent convection in the solar envelope. The widely used mixing-length theory (MLT) oversimplifies the treatment of approaching isotropy for anisotropic turbulence and the dissipation of the turbulence. We shall investigate the effects of these factors on solar models.

Dynamic equations for steady state turbulent convection can be found in Hinze (1975), Canuto (1992, 1997), Xiong (1989), and Xiong et al. (1997). In order for these equations to be solvable, some simplifications should be made. The dissipation of the turbulent kinetic energy is often modeled by Hinze (1975) and Hossain and Rodi (1982). The dissipation of the velocity-temperature correlation needs to be considered more carefully for stellar turbulent convection. There are two dissipation sources: the molecular viscosity decreases the fluctuating velocity shearing, while the thermal conduction flattens the fluctuating temperature stratification. The combination of these effects restricts the fluctuations of the velocity and temperature, leading to a dissipation of the velocity-temperature correlation. Two time scales are involved therefrom: $\tau_{k} \sim \frac{k}{\varepsilon}$ is the time that the kinetic energy is exhausted and $\tau_{h} \sim \frac{\bar{\rho} c_{P}}{\lambda} \frac{k^{3}}{\varepsilon^{2}}$ the time that eddies lose all extra heat to their surroundings. Based on the above arguments we propose that the dissipation of the velocity-temperature correlation is proportional to the product of itself and the sum of the above two time scales. Based on similar arguments, it is plausible to assume the dissipation of the self-correlation of the temperature fluctuation is of similar form to that of the velocity-temperature correlation. For the redistribution term containing the pressure-strain correlation, Rotta's proposal of "return to isotropy" is adopted (Rotta 1951).

Applying the above turbulence model to a stellar convection zone, and assuming that the mean-flow velocity is zero while all other physical quantities have only radial component and are only the function of the radius in the star, we obtain an equation to determine the temperature gradient of the convection 
zone:

$$
\begin{aligned}
& \left(f-\frac{C_{T} P_{\mathrm{e}}^{-2}}{D+\frac{1}{C_{\theta}}}+\sqrt{\left(f-\frac{C_{T} P_{\mathrm{e}}^{-2}}{D+\frac{1}{C_{\theta}}}\right)^{2}+\frac{8 C_{T} P_{\mathrm{e}}^{-2}}{\left(D+\frac{1}{C_{\theta}}\right)^{2}}\left[D f-C_{T}(1-f)\right]}\right)^{3} \\
= & \frac{64 C_{T}^{3} P_{\mathrm{e}}^{-4}}{\left(D+\frac{1}{C_{\theta}}\right)^{3}}(1-f)
\end{aligned}
$$

where

$$
P_{\mathrm{e}}=-\frac{\bar{\rho} g_{r} l^{2} c_{P}}{\lambda} \sqrt{\frac{\bar{\rho} \beta}{\bar{P}}\left(\nabla_{r}-\nabla_{s}\right)}
$$

In Eq. (1), $D$ describes the degree of the anisotropy of the turbulence, $C_{T}$ and $C_{\theta}$ are two parameters to determine the dissipation of the turbulence. Equation (1) is of similar form as the standard MLT cubic equation (Kippenhahn 1962). The solutions of Eq. (1) are compared with that of the standard MLT in Fig. 1.

\section{Applications to Solar Models}

We computed three models, MS1, MS2, and MS3, with different choices of the turbulent parameters $C_{T}, C_{\theta}$, and $D$. The main results are summarized in Table 1. It can be seen that the solar models resulted from the improved MLT are not significantly different from the standard solar model (SSM). The most difference appears in the convection parameter $\alpha$, which is the ratio of the mixing length to local pressure scale height. The use of the improved MLT greatly decreases the value of the convection parameter $\alpha$. When $D=1.0, C_{T}=3.0$, and $C_{\theta}=1.25, \alpha$ is found to be 0.977 , which is much smaller than the standard solar model's value of 1.638. When the anisotropic degree of the turbulence increases from 1.0 to 2.0 , we can expect a corresponding increase of the convective energy transport efficiency, and the convection parameter $\alpha$ is indeed found to be an even smaller value of 0.708 . Another effect, to decrease the dissipation of the turbulence, can also enhance the convective energy transport. It is found that taking the convection parameter $\alpha$ to be 0.433 can readily reproduce the present solar parameters when the dissipation parameter $C_{T}$ decreases from 3.0 to 1.5. These results indicate that the approximations introduced in the present turbulence model work consistently and are reasonable improvements to the standard MLT.

The frequencies of the p-mode oscillations for the spherical harmonic index $l=0,1,2,3$ are computed based on the considered solar models. Figure 2 shows the differences of these frequencies with respect to the observed values. The dotted lines that represent the results of the MS3 almost coincide with the solid lines that represent those of the SSM, while the results of the MS1 and MS2 are almost identical. But the frequencies of the MS1 and MS2 are about $1 \mu \mathrm{Hz}$ lower than that of the SSM and MS3 in the low frequency section, and are in better agreement with the observations. These results imply that the dissipation of the turbulence in the solar convection zone should be fairly strong, while the anisotropic degree of the turbulence plays a less significant role in the structure of the solar convection zone. 
Table 1. Properties of the Standard and Improved Solar Models

\begin{tabular}{lllll}
\hline Models & SSM & MS1 & MS2 & MS3 \\
\hline \hline Turbulence & & $D=1.0$ & $D=2.0$ & $D=2.0$ \\
Parameter & & $C_{T}=3.0$ & $C_{T}=3.0$ & $C_{T}=1.5$ \\
\hline$\alpha$ & 1.6377 & 0.9769 & 0.7081 & 0.4330 \\
\hline$X_{0}$ & 0.7049 & 0.7049 & 0.7049 & 0.7049 \\
\hline$X_{\mathrm{c}}$ & 0.348480 & 0.348473 & 0.348473 & 0.348482 \\
\hline$T_{\mathrm{c}}\left(\times 10^{6} \mathrm{~K}\right)$ & 15.69 & 15.69 & 15.69 & 15.69 \\
\hline$\rho_{\mathrm{c}}\left(\mathrm{g} / \mathrm{cm}^{3}\right)$ & 153.2 & 153.2 & 153.2 & 153.2 \\
\hline$M_{\mathrm{bc}}\left(M_{\odot}\right)$ & 0.979 & 0.979 & 0.979 & 0.979 \\
\hline$R_{\mathrm{bc}}\left(R_{\odot}\right)$ & 0.724 & 0.724 & 0.724 & 0.724 \\
\hline$T_{\mathrm{bc}}\left(\times 10^{6} \mathrm{~K}\right)$ & 2.122 & 2.122 & 2.122 & 2.122 \\
\hline$\Phi_{\mathrm{Cl}}(\mathrm{SNUs})$ & 7.70 & 7.70 & 7.70 & 7.70 \\
\hline$\Phi_{\mathrm{Ga}}(\mathrm{SNUs})$ & 127.50 & 127.50 & 127.50 & 127.50 \\
\hline$\# C_{\theta}=1.25$ & & & &
\end{tabular}

\section{Summary}

We computed some solar models based on an improved mixing-length theory which includes the effects of anisotropy and dissipation of the turbulence. It is found that the improved MLT greatly decreases the value of the convection parameter $\alpha$, and the dissipation of the turbulence in the solar convection zone should be fairly strong, while the anisotropic degree of the turbulence plays a less significant role in the solar convection zone. This work is sponsored by the NSFC through project number 19625306 and 19833040, and National Key Fundamental Research Project "Formation and Evolution of Galaxies".

\section{References}

Canuto, V.M. 1992, ApJ, 392, 218

Canuto, V.M. 1997, ApJ, 482, 827

Hinze, J.O. 1975, Turbulence, Second Edition (New York: McGraw-Hill)

Hossain, M.S., \& Rodi, W. 1982, in Turbulent Buoyant Jets and Plumes, ed. W. Rodi, The Science and Applications of HMT, Vol. 6, p.121

Kippenhahn, R. 1962, Proc. Int. School of Physics "Enric Fermi", XXVIII, p.330

Rotta, J.C. 1951, Z. für Physik, 129, 547

Xiong, D.R. 1989, A\&A, 209, 126

Xiong, D.R. Cheng, Q.L., \& Deng, L., 1997, ApJS, 108, 529 

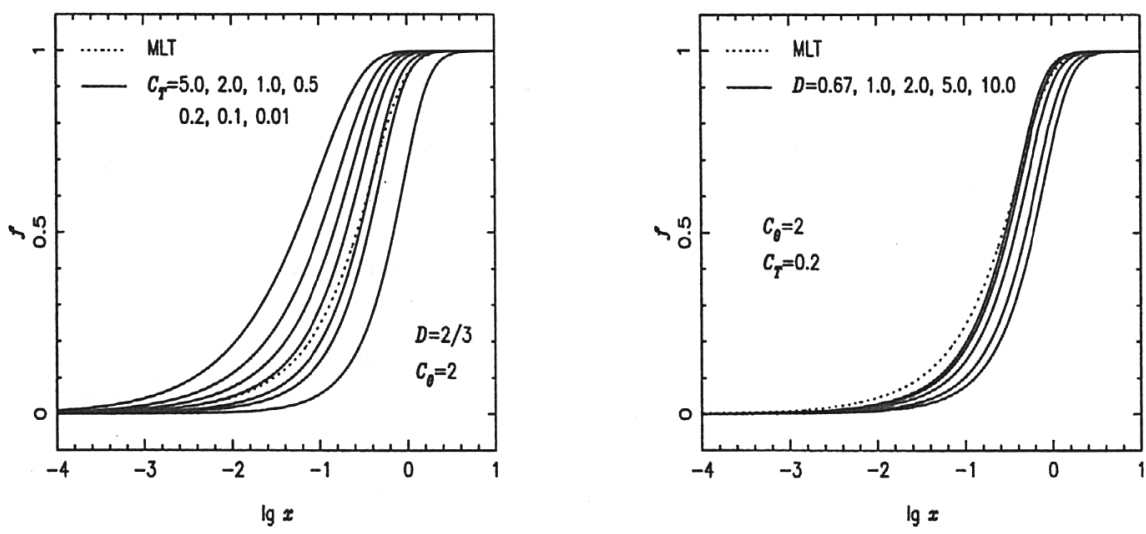

Figure 1. Comparisons of the efficiency of convection energy transport under different turbulence parameters. The MLT value is given by dotted lines. $x$ is related to $P_{\mathrm{e}}$ by $x=\sqrt{\frac{C_{T}}{8 D}} \frac{1}{P_{\mathrm{e}}}$
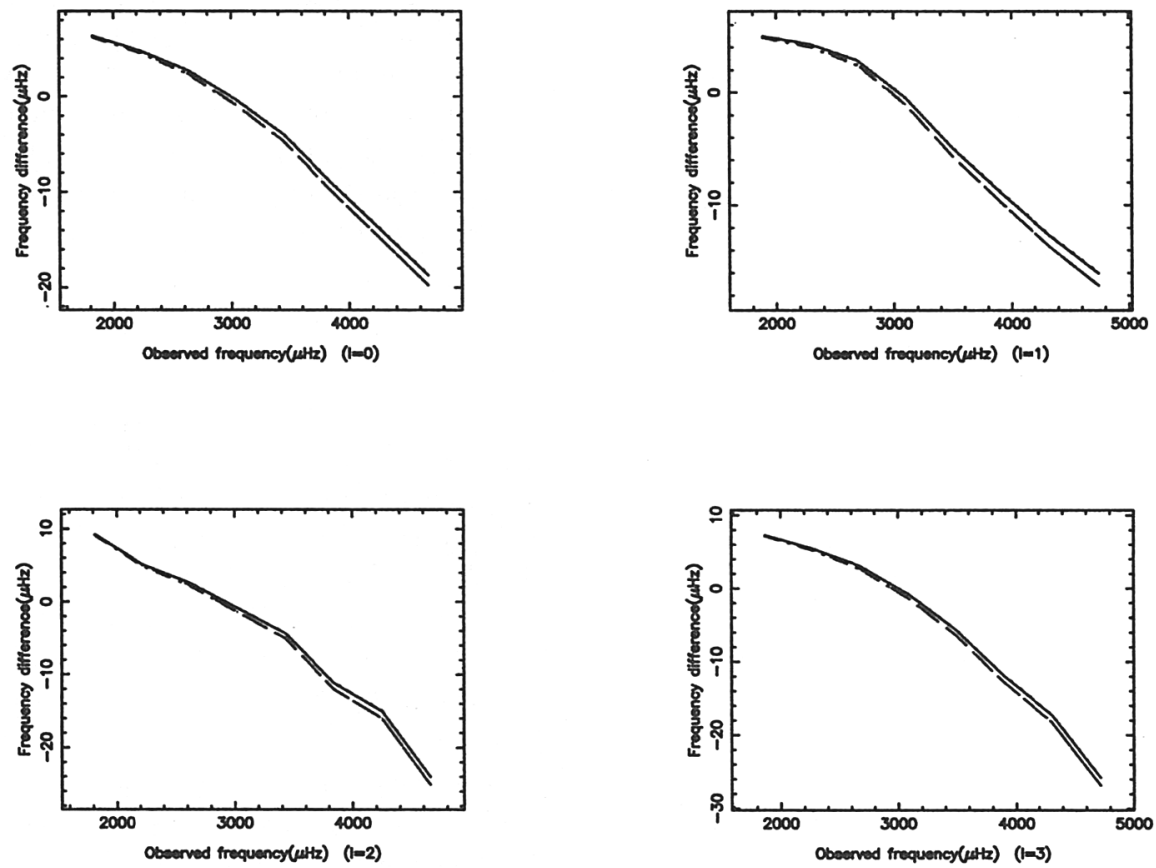

Figure 2. Differences of the p-mode frequencies of computed solar models with respect to the observed values. Solid and dotted lines are for SSM and MS3, respectively, and dashed lines for MS1 and MS2 